\title{
The effect of storage temperature on the metabolic profiles derived from chicken eggs
}

by Johnson, A.E., Sidwick, K.L., Pirgozliev, V.R., Edge, A. and Thompson, D.F.

Copyright, Publisher and Additional Information: This is the author accepted manuscript. The final published version (version of record) is available online via Elsevier.

This version is made available under the CC-BY-ND-NC licence:

https://creativecommons.org/licenses/by-nc-nd/4.0/legalcode

Please refer to any applicable terms of use of the publisher

DOI: https://doi.org/10.1016/j.foodcont.2019.106930

\section{Harper Adams} University 


\title{
The Effect of Storage Temperature on the Metabolic
}

\section{Profiles Derived from Chicken Eggs}

\author{
Amy E. Johnson ${ }^{a}$, Kate L. Sidwick ${ }^{b}$, Vasil R. Pirgozliev' ${ }^{c}$, Anthony Edge ${ }^{d}$, and David \\ F. Thompson*e
}

a School of Chemical and Physical Sciences, Keele University, Keele, Staffordshire, ST5 5BG, United

Kingdom. Email: a.johnson1@keele.ac.uk

b School of Chemical and Physical Sciences, Keele University, Keele, Staffordshire, ST5 5BG, United Kingdom. Email: k.sidwick@keele.ac.uk

\begin{abstract}
c The National Institute of Poultry Husbandry, Harper Adams University, Newport, Shropshire, TF10 8NB, United Kingdom. Email: vpirgozliev@harper-adams.ac.uk

d Department of Chemistry, Liverpool University, Crown Street, Liverpool, L69 7ZD, United Kingdom. Email: Tony.Edge@liverpool.ac.uk
\end{abstract}

$\mathrm{e}^{*}$ School of Chemical and Physical Sciences, Keele University, Keele, Staffordshire, ST5 5BG, United Kingdom. Email: d.f.thompson@keele.ac.uk

\section{Abstract}

Metabonomic profiling, using High Performance Liquid Chromatography Quadrupole Timeof-Flight Mass Spectrometry (HPLC-Q-ToF-MS), was adopted in this study to uncover differences in the small molecule profiles of egg yolks, between two sets of six eggs that were stored at $5^{\circ} \mathrm{C}$ and $23^{\circ} \mathrm{C}$ for five weeks. Choline, a compound that has previously been shown to have potential use as a biomarker of egg age, was observed to have a significantly lower abundance in the yolks of eggs that were stored at $5^{\circ} \mathrm{C}$ for five weeks compared to eggs stored at $23^{\circ} \mathrm{C}$ for the same length of time. A follow-up targeted study observed that the previously discovered trend of increasing choline concentration in egg yolk, with increasing egg age, is not repeatable when the eggs are stored at $5^{\circ} \mathrm{C}$ rather than $23^{\circ} \mathrm{C}$. It 
concluded that, by refrigerating eggs at $5^{\circ} \mathrm{C}$, the accurate prediction of egg age using choline as a biomarker can be prevented.

\section{Keywords}

Metabonomics, Chromatography, Mass Spectrometry, Eggs, Fraud, Choline

\section{Introduction}

Food fraud is the act of misleading, or misinforming consumers regarding the food products that they purchase and consume. It is an intentional act of deception, and is usually performed in order to achieve some financial gain (Spink \& Moyer, 2011; Spink, Ortega, Chen, \& Wu, 2017). It is not a modern problem, but due to the expansion of food supply chains across the globe over more recent years, the effects of food fraud have become more far-reaching, and the impact of food fraud on consumers and food businesses has increased (Manning \& Soon, 2014; Spink \& Moyer, 2011; Spink et al., 2017). Because of this widening and increasing impact of food fraud, consumers are becoming more aware (Hong et al., 2017; Spink \& Moyer, 2011), and their trust in food businesses is negatively affected (Spink et al., 2017; Tähkäpää, Maijala, Korkeala, \& Nevas, 2015). This causes problems for food businesses, due to a lack of sales, which also affects the government through the loss of Value Added Tax (Tähkäpää et al., 2015). Other economic implications come from the additional costs of product recalls and fraud investigations (Spink et al., 2017).

Metabonomics is the study of metabolic profile changes, in organic tissues and biofluids, in response to internal and external factors (Tang \& Wang, 2006; Wilson et al., 2005). It has been employed in a variety of different studies, ranging from drug discovery (Goodacre, Vaidyanathan, Dunn, Harrigan, \& Kell, 2004), to environmental studies (Moco, Bino, De Vos, \& Vervoort, 2007), as well as food authentication studies (Rubert, Zachariasova, \& Hajslova, 2015). Non-targeted metabonomic studies are useful when little is known about which 
metabolic pathways and compound classes may be affected by the variable under investigation, as they aim to gain as much information about the small molecule profiles of samples, and how they change, as possible (Gika, Theodoridis, Plumb, \& Wilson, 2014). Non-targeted studies have the potential to discover a particular compound, or class of compounds, that can act as a biomarker for the particular factor under investigation. If this is successful, then a follow-up targeted study may be carried out to focus the analysis on the potential biomarker in a more robust experiment, to see if the results are replicable, and to gain more quantitative information about the marker.

European Union legislation states that the sell-by dates of shell eggs is 3 weeks post lay, and the use-by date is 4 weeks post lay (“Regulation (EC) 853/2004," 2004). However, as there is no way for the consumer to confirm whether or not the dates on the labels match up with the ages of the eggs themselves, it would be easy for eggs to be incorrectly labelled with false dates, misleadingly extending the time before they reach their sell-by and use-by dates. This mislabelling of eggs with incorrect dates is an example of food misrepresentation, which is the false description or advertisement of produce and is one of the most common forms that food fraud takes (Spink et al., 2017).

Previous research using a non-targeted, and follow up targeted, metabonomic study, has discovered choline to be a potential biomarker of egg age (Johnson, Sidwick, Pirgozliev, Edge, \& Thompson, 2018), with potential capabilities of detecting cases of fraud due to sellby and use-by date mislabelling. Choline was found to increase in concentration in egg yolk with increasing egg age, up to twelve weeks of egg storage at $23^{\circ} \mathrm{C}$. However, choline is present in its free form due to the catabolism of phosphatidylcholines by phospholipases (Lockman \& Allen, 2002; Zeisel \& Da Costa, 2009); as the phosphatidylcholine content of egg yolk decreases throughout an increasing storage time of eggs (Wang et al., 2017), the egg yolk choline concentration increases (Johnson et al., 2018). If eggs were stored refrigerated at $5^{\circ} \mathrm{C}$, then the rate of catabolism of phosphatidylcholines would decrease due to a reduction in the rate of phospholipase-catalysed reactions caused by the lower 
temperature, and so the previously observed increase in choline concentration with increasing egg age may not be replicable in these refrigerated conditions.

This paper aims to investigate whether the storage of eggs for five weeks at two different temperatures, $5^{\circ} \mathrm{C}$ and $23^{\circ} \mathrm{C}$, results in different metabolic profiles in the yolks of the eggs. It will then investigate through a targeted study, whether the storage of eggs for twelve weeks at $5^{\circ} \mathrm{C}$ results in the same observed increase in choline concentration in egg yolk as in previous studies, or whether the lower storage temperature inhibits this trend, and thus prevents the accurate prediction of egg age based on yolk choline concentration, and therefore obstructs the detection of fraud.

\section{Materials and Methods}

This research employs a workflow, from metabolite extraction to statistical analysis, that has been developed by the authors, and carried out in previous studies (Johnson et al., 2018)(Johnson, Sidwick, Pirgozliev, Edge, \& Thompson, 2019).

\subsection{Materials}

Methanol (HPLC, isocratic grade) and dichloromethane (stabilized with 0.002\% 2-methyl-2butene) were purchased from VWR (Radnor, PA). Ultra-pure water $(18.2 \mathrm{M} / \mathrm{cm})$ was purified in-house using a Milli-Q system from Elga (High Wycombe, U.K.). Ammonium acetate and formic acid (90\%, laboratory reagent grade) were purchased from Fisher Scientific (Loughborough, U.K). API-TOF reference-mass solution and ESI-L lowconcentration tuning mix were purchased from Agilent Technologies (Santa Clara, CA).

\subsection{Sample Collection (non-targeted study)}

Eggs were collected for the non-targeted study from The National Institute of Poultry Husbandry, Harper Adams University, U.K. Laying hens were of the Hy-line brown breed 
and were 21 weeks old at the point of lay. Birds were kept in enriched cages, of dimensions $1205 \mathrm{~cm} \times 50 \mathrm{~cm}$ floor space, and $67 \mathrm{~cm}$ height, with eight birds per cage. Six eggs were stored at $5^{\circ} \mathrm{C}$, and six were stored at $23^{\circ} \mathrm{C}$ for five weeks, at which point an organic liquid extraction was carried out.

\subsection{Sample Collection (targeted study)}

Eggs were collected for the targeted study from Oaklands Farm Eggs Ltd, U.K. Laying hens were of the Hy-line Brown breed and were 46 weeks old at the point of lay. Birds were kept in enriched cages, with 80 birds per cage. Eggs were stored at $5^{\circ} \mathrm{C}$ and an organic liquid extraction was carried out on six eggs every seven days; ranging from fresh eggs (week 0 ) to eggs stored for twelve weeks, resulting in a total of thirteen time points.

\subsection{Metabolite Extraction}

One metabolite extraction per yolk, for each of the six eggs at each time point, was carried out as described in (Johnson et al., 2018). As egg albumen does not contain as many compounds as the yolk (Li-Chan \& Kim, 2008), the egg yolk was the focus of the analysis. A stainless steel egg separator was used to separate the egg yolk and albumen, and approximately $50 \mathrm{mg}$ of yolk was weighed out. A ratio of $1 \mathrm{~mL}$ per $50 \mathrm{mg}$ yolk of organic extraction solvent mixture (dichloromethane:methanol, 3:1) was then added, and the sample was centrifuged using a Fisherbrand accuSpin Micro 17 microcentrifuge, at 16,100 rcf for 20 minutes. The supernatant was removed and the sample extract dried under ambient conditions. An equal volume of methanol was then added to the dried extract, which was then briefly vortexed for approximately five seconds, and stored at $-80^{\circ} \mathrm{C}$ prior to analysis.

\subsection{Quality Control}

A Quality Control (QC) sample for the non-targeted study was created from aliquots of equal volume from all sample extracts, in accordance with published guidelines (Sangster, Major, 
Plumb, Wilson, \& Wilson, 2006). This QC sample was injected and analyzed ten times immediately prior to the samples, in order to condition the column ready for the analysis. Further injections of this QC sample took place between every six samples during the analytical sequence in order to monitor instrumental drift. For the targeted analysis, a 0.5 $\mu \mathrm{g} / \mathrm{mL}$ solution of choline chloride in methanol was used as a QC sample and was injected and analyzed between every six or seven samples throughout the analysis. In order to prevent any instrumental bias from affecting the results, the sequence of injections was randomized prior to analysis. Each sample was injected and analysed once during the analytical sequence.

\subsection{Chromatographic Parameters}

A Thermo Scientific Accucore RP-MS column (100 mm x $2.1 \mathrm{~mm}, 2.6 \mu \mathrm{m}$ particle size), kept at a temperature of $40^{\circ} \mathrm{C}$, was used for chromatographic separation, on an Agilent Technologies 1260 Infinity Binary HPLC system.

\subsubsection{Non-targeted Chromatographic Parameters}

The sample injection volume was $5 \mu \mathrm{L}$, the flow rate used was $0.3 \mathrm{~mL} / \mathrm{min}$, and a needle wash in methanol was carried out during each injection. Solvent $(A)$ was $0.1 \%$ formic acid and $5 \mathrm{mM}$ ammonium acetate, and solvent $(\mathrm{B})$ was methanol with $0.1 \%$ formic acid and $5 \mathrm{mM}$ ammonium acetate. The solvent ratio started at $75 \%(\mathrm{~B})$, and then increased up to $81 \%$ (B) over the first 20 minutes, then up to $90 \%$ (B) in 1 minute, at which point it was held for 10 minutes. It was then increased to $100 \%$ (B) in 30 minutes, held at $100 \%$ (B) for 20 minutes, then returned to starting conditions over the final 4 minutes. The total analysis time was 85 minutes, plus a post time of 5 minutes to allow the instrument to equilibrate prior to the next sample injection.

\subsubsection{Targeted Chromatographic Parameters}


The injection volume of samples was $1.5 \mu \mathrm{L}$, the flow rate was $0.2 \mathrm{~mL} / \mathrm{min}$, and a needle wash of methanol was again carried out during each injection. Solvents $(A)$ and $(B)$ were the same as in the non-targeted analysis. The solvent gradient started at 5\% (B) and was held for one minute, before increasing to $10 \%(B)$ in 0.1 minutes. It was then decreased to $5 \%(B)$ in 1.4 minutes and held for 0.5 minutes. External standards of choline chloride in methanol were analyzed in a range of concentrations: $0.2,0.3,0.4,0.5,0.6,0.7,0.8 \mu \mathrm{g} / \mathrm{mL}$. Each standard was injected and analysed three times, both before and after the samples, in order to calculate the mean and Relative Standard Deviation percentages (RSD\%s) for the peak areas of each of the standards for both the standard and drift calibration curves.

\subsection{Mass Spectrometry (MS) Parameters}

The HPLC was coupled to an Agilent Technologies 6530 Accurate-Mass Quadruple-Time-ofFlight mass spectrometer, with a mass resolution of $>20,000$ FWHM. Analysis was carried out in positive ionization mode, and an electrospray ion source was used. No analysis was carried out in negative ionization mode, as the authors discovered through previous analysis that very few molecules in the organic extract of egg yolk are ionized under negative ionization, resulting in much smaller data sets than those obtained through analysis in positive ionization mode. The drying gas temperature was $300^{\circ} \mathrm{C}$ and had a flow rate of 8 $\mathrm{L} / \mathrm{min}$. The capillary voltage, fragmentor voltage and skimmer voltage were $3500 \mathrm{~V}, 175 \mathrm{~V}$, and $65 \mathrm{~V}$ respectively and the nebulizer pressure was $35 \mathrm{psi}$. A mass range of $100-1000 \mathrm{~m} / \mathrm{z}$ was used for the non-targeted study and $50-200 \mathrm{~m} / \mathrm{z}$ for the targeted study. The system was calibrated prior to analysis, using the ESI-L low concentration tuning mix, in order to ensure the mass accuracy. The mass accuracy was maintained throughout the analysis, using the API-TOF reference mass solution.

\subsection{Non-Targeted Statistical Analysis}


Raw data was pre-processed using XCMS Online, which produced a feature table, with each feature/compound represented by its $\mathrm{m} / \mathrm{z}$ and retention time, giving the peak areas for each compound in all samples. This table was then transferred into Microsoft Exce/ where RSD\%s were calculated for each compound based on the QC samples. Those with a RSD\% $>30 \%$ were removed from further data analysis, in alignment with published guidelines (Theodoridis, Gika, Want, \& Wilson, 2012). An F-test was carried out on all remaining compounds, and then t-tests were carried out for either equal or unequal variances, depending on the results of the F-test, to see which compounds in egg yolks were significantly different between eggs stored at $5^{\circ} \mathrm{C}$ and $23^{\circ} \mathrm{C}$ for five weeks. The significance level was set at $p<0.01$ and all features with $p>0.01$ were removed. Principal Component Analysis (PCA) was carried out, including all remaining compounds in the analysis, using an Excel Multivariate Analysis add-in. The PCA included six principal components, and the data was standardized prior to analysis. Scores plots were then produced to display the variation between sample sets. PC1 loadings (as these describe the most variance within the data) were used to rank the features and the top 100 with the highest loadings were taken for further analysis. Any duplicates or isotopes were removed from this top 100 and the statistical significance of the remaining features was then confirmed using Agilent Technologies MassHunter Qualitative Analysis to analyse the raw data. Extracted Ion Chromatograms were produced for each feature and the peak areas of the integrated peaks were used to carry out F-tests and t-tests as done previously. The significance level for the raw data was set at $\mathrm{p}<0.05$, as this had not been pre-processed so more allowance was given for statistical significance. Attempts were then made to identify the compounds that were still found to be statistically significant from the raw data.

\subsubsection{Feature Identification}

Potential molecular formulas were predicted for these compounds, using Agilent Technologies' MassHunter Qualitative Analysis software, based on the monoisotopic mass, isotope abundance, and isotopic peak spacing in the mass spectra. All formulas with a 
likelihood score of 95 and above were searched on METLIN to find potential metabolite matches. The sample mass spectra were compared with the mass spectra of potential matches on METLIN to see if they could be putatively identified based on comparable mass spectra.

\subsection{Targeted Statistical Analysis}

MassHunter Qualitative Analysis was used to produce EICs of choline from the raw data, for the external standards and the samples. Standard and drift calibration curves were produced, based on the mean peak areas of the integrated peaks in the EICs of choline for the external standards. The accuracy of the data was measured by calculating Relative Errors (REs), and the precision of the data was measured by calculating RSD\%s. Both were within accepted limits, according to the Food and Drug Administration (FDA) guidelines (FDA, 2018). The equation of the standard curve was then used to calculate the concentration of choline in each sample of egg yolk, based on the peak areas of the integrated peaks in the EICs of choline in the samples. A Levene's test for equality of variances was carried out, which showed equal variances in choline concentration between the sample sets, followed by ANOVA to test for a statistical significance of choline concentration when comparing eggs stored for different lengths of time. A post-hoc Tukey test was then carried out to see which sets of eggs of different ages were significantly different to each other.

\section{Results and Discussion}

\subsection{Non-targeted Study}

\subsubsection{Metabolite Profiling}

The metabolite profiles of the yolks of eggs that have been stored for five weeks at $5^{\circ} \mathrm{C}$ show a slight, but visible difference to those of eggs that have been stored for the same 
length of time at $23^{\circ} \mathrm{C}$, as can be seen in Figure 1.

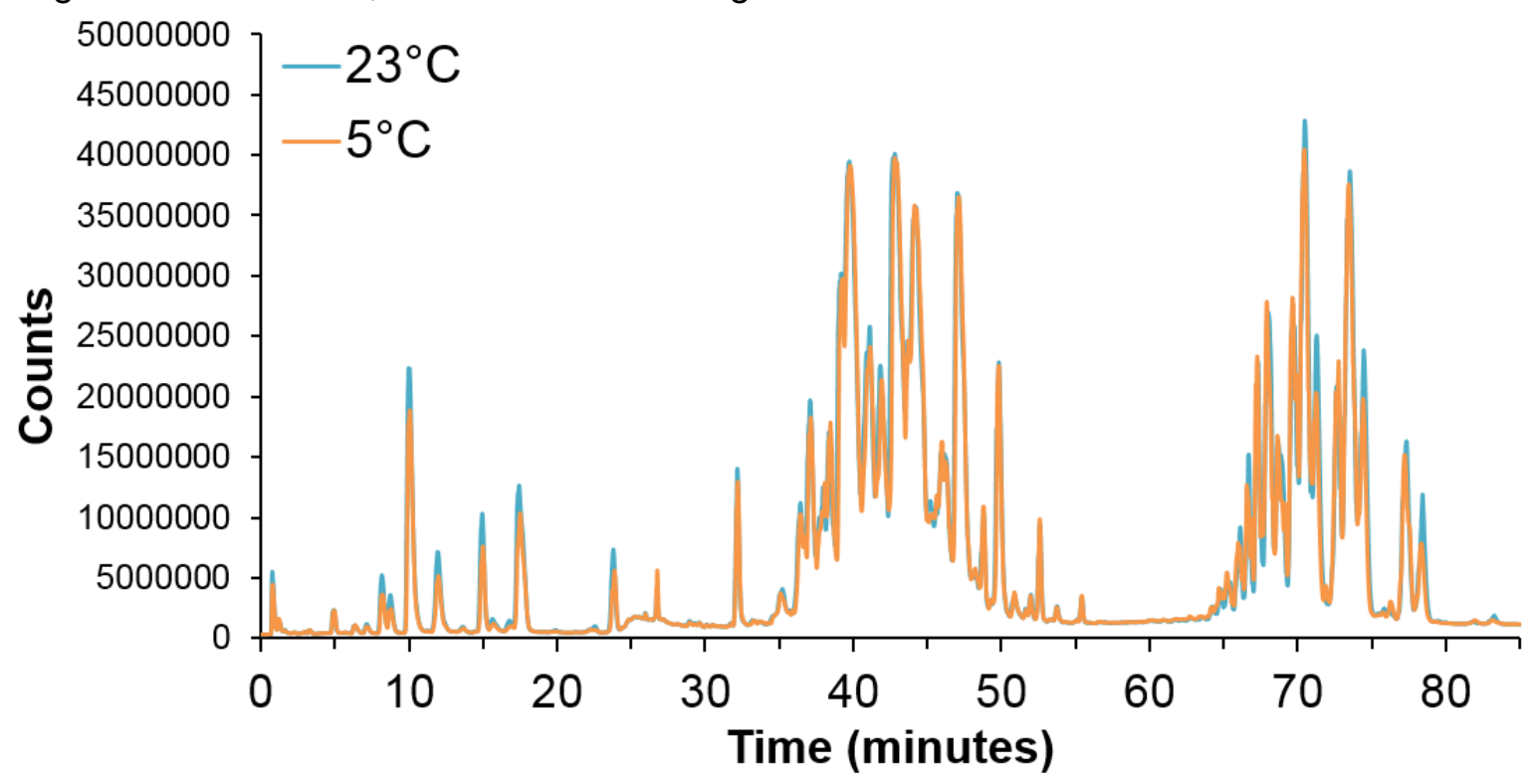

Figure 1. Figure 1. Overlaid Total Ion Chromatograms for eggs stored at $5^{\circ} \mathrm{C}$ and $23^{\circ} \mathrm{C}$ for five weeks, based on median values of retention time and detector response

There is a higher intensity for most chromatographic peaks in the profiles of eggs stored at $23^{\circ} \mathrm{C}$, however there are also some peaks that seem to be of a higher intensity in the profiles of eggs that were stored at $5^{\circ} \mathrm{C}$. This is likely because some peaks will correspond to compounds that are catabolic products of other compounds, such as choline, monoglycerides, and diglycerides, and so will be of a higher abundance in eggs stored at $23^{\circ} \mathrm{C}$ because the rate of catabolism due to enzyme-catalysed reactions is higher at higher temperatures. However, other peaks will correspond to the compounds that are breaking down and being catabolised throughout the storage period, such as phospholipids e.g. phosphatidylcholines and phosphatidylethanolamines, and so these will be of a higher abundance in eggs stored at $5^{\circ} \mathrm{C}$ because the rate of catabolism is slower at lower temperatures.

\subsubsection{Multivariate Statistics}


The scores plot in Figure 2, which

was produced following PCA, shows

a clear separation between eggs that

were stored at $5^{\circ} \mathrm{C}$ and those that

were stored at $23^{\circ} \mathrm{C}$. The $\mathrm{QC}$ samples

are grouped closely together,

indicating that the analysis was robust

and that true biological differences

- QCs
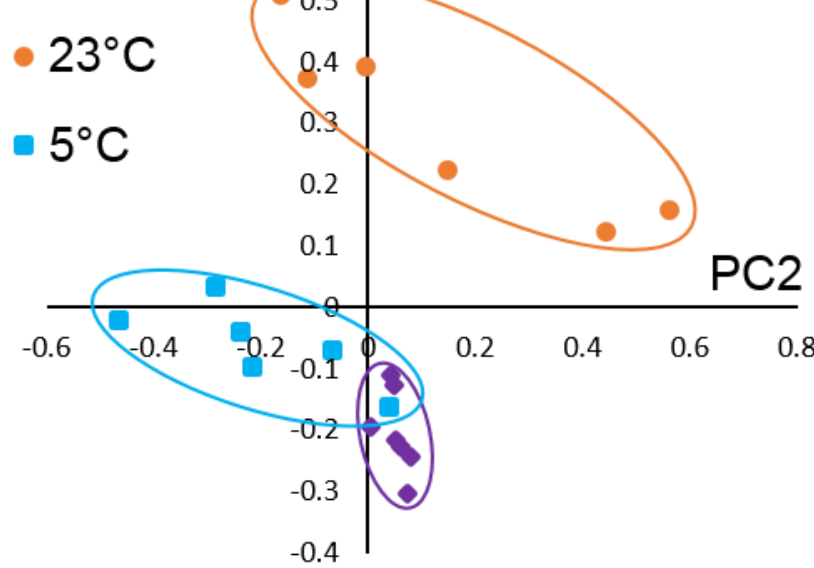

are responsible for the separation

between the two sample sets, as

Figure 2. PCA scores plot of PC2 vs PC4 for features with RSD\% $<30 \%$ and $P<0.01$, showing separation between eggs that were stored for five weeks at $5^{\circ} \mathrm{C}$ and $23^{\circ} \mathrm{C}$

opposed to instrumental drift occurring throughout the analysis. Although most separation between the sets of eggs stored at different temperatures is shown by PC4, this principal component is actually only responsible for describing $0.003 \%$ of the variation in the data, which shows just how subtle these metabonomic differences are between the eggs stored at the two different temperatures.

\subsubsection{Compound Differences}

After removing any duplicates and isotopes from the top 100 compounds based on PC1 loadings, 49 features still remained. Of these 49 features, 29 were still found to be statistically significant following confirmatory t-tests based on the raw data. Table 1 shows these compounds, represented by their $\mathrm{m} / \mathrm{z}$ and retention times, and their mean peak areas for eggs kept at each storage temperature, their RSD\%s, and p-values based on initial ttests following data pre-processing. One of these compounds, $\mathrm{m} / \mathrm{z} 331.2856$, was successfully putatively identified through METLIN as 1-monopalmitin, and was found to be of a higher abundance in the yolks of eggs that were stored at $23^{\circ} \mathrm{C}$ for five weeks, compared to those that were stored at $5^{\circ} \mathrm{C}$. Most of the lower molecular weight compounds, which are likely to correspond to compounds that are products of catabolism, such as monoglycerides and diglycerides, are of a higher abundance in the yolks of eggs that were stored at $23^{\circ} \mathrm{C}$ 
compared to eggs that were stored at $5^{\circ} \mathrm{C}$. This is what would be expected, as the lower storage temperature will have slowed down the catabolism of compounds, therefore slowing down the rate of release of compounds that are products of catabolism, resulting in a lower abundance of these compounds in the yolks of eggs that were stored at this temperature. Most of the compounds that show a higher abundance in the yolks of eggs that were stored at $5^{\circ} \mathrm{C}$ for five weeks are the higher molecular weight compounds, which are likely to correspond to compounds that undergo catabolism, such as phospholipids. This again was expected, as the lower storage temperature will have slowed down the catabolism of these compounds, resulting in the yolks of eggs stored at the higher storage temperature having a lower abundance of these compounds, as they would be broken down more quickly at this temperature.

The compound $m / z 104.1072$ has been identified in previous work as choline (Johnson et al., 2018) and has been observed to increase in concentration in egg yolk over an increasing storage time at $23^{\circ} \mathrm{C}$. Figure 3 shows the difference in abundance of choline in egg yolk between eggs stored at $5^{\circ} \mathrm{C}$ and $23^{\circ} \mathrm{C}$.

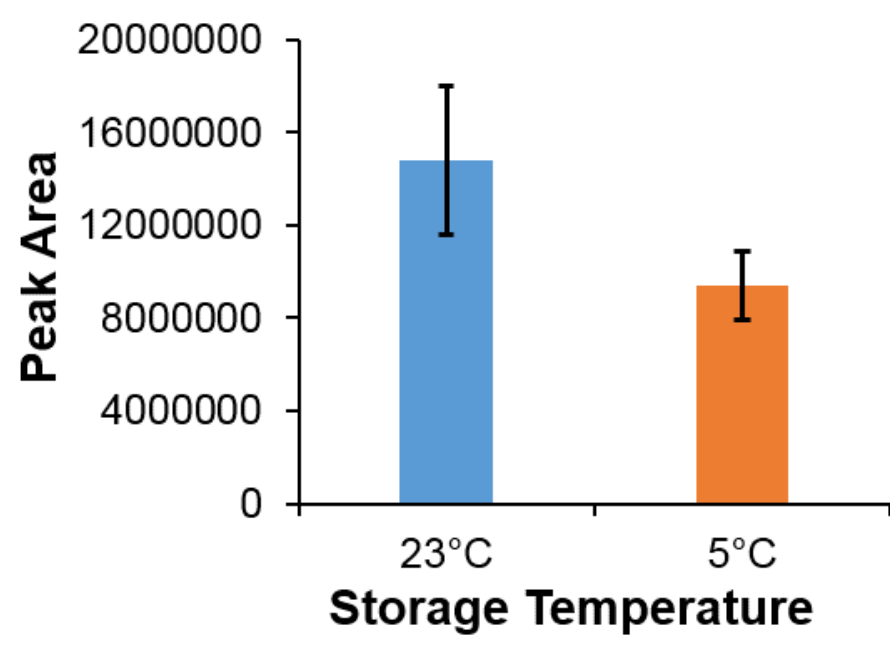

Figure 3. Bar chart showing the difference in abundance of choline in egg yolk between eggs stored at $5^{\circ} \mathrm{C}$ and $23^{\circ} \mathrm{C}$ for five weeks, based on mean peak areas. Error bars represent $+/$ - 1 standard deviation.
Figure 3 shows that choline abundance in egg yolk is significantly lower in eggs stored at $5^{\circ} \mathrm{C}$ compared to $23^{\circ} \mathrm{C}$, which indicates that the storage of eggs at a lower temperature affects the rate of increase of choline abundance. Lower storage temperatures result in a lower rate of catabolism by enzymecatalysed reactions. As choline is

formed from the catabolism of phosphatidylcholines by phospholipases (Lockman \& Allen, 2002; Zeisel \& Da Costa, 2009), the rate of choline production will be decreased, resulting in 
a lower choline abundance in egg yolk after five weeks of storage, compared to when eggs are stored at higher temperatures.

\subsection{Targeted Study}

The standard and drift calibration curves both had $R^{2}=0.99$. The REs for the standards were all less than $7 \%$ for the standard curve and less than $15 \%$ for the drift curve. The RSD $\%$ s were all less than $13 \%$ for the standard curve and less than $14 \%$ for the drift curve. The RSD\% for the QC sample injected throughout the analysis was found to be $3.6 \%$.

The results of the ANOVA showed that there was a significant difference in choline concentration between eggs stored for different lengths of time at $5^{\circ} \mathrm{C}$, with $\mathrm{p}=0.001$. However, the Tukey test revealed that there were only significant differences between four pairwise comparisons of egg age. Eggs that were stored for one week showed significant differences in choline concentration compared to eggs that were stored for six, eight, ten and twelve weeks, which suggests that there may not be a trend in the changing abundance of choline with increasing storage time at $5^{\circ} \mathrm{C}$. This is confirmed by the graph in Figure 4 , which shows an erratic change in compound abundance between the yolks of eggs that were stored for increasing lengths of time.

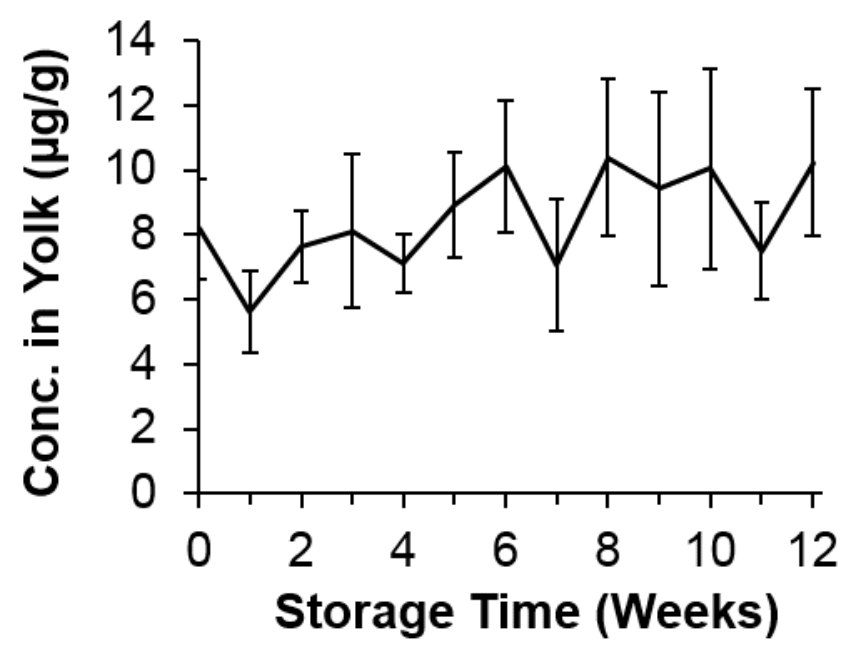

As can be seen from Figure 4, there was a decrease in choline concentration over the first week of storage, resulting in the lowest concentration of choline observed in this study, followed by an increase the following week. This explains why only the eggs that were stored for one week show significant differences in choline concentration compared to some other sets of 
eggs that were stored for longer periods of time. Although there is some statistical significance in the changing concentration of choline between the yolks of eggs that were stored for increasing lengths of time, as this change in concentration is highly erratic and does not follow a general trend over the twelve-week storage period, it suggests that storing eggs at lower temperatures prevents the increase in choline concentration which was previously observed when eggs were stored at $23^{\circ} \mathrm{C}$. This is likely to be due to a reduction in the rate of phospholipase-catalysed reactions caused by the lower temperature, resulting in the production of fewer choline molecules from phosphatidylcholines. This lack of increase in yolk choline concentration with increasing egg age, when eggs were stored at $5^{\circ} \mathrm{C}$ for up to five weeks, corroborates what was observed from the non-targeted study, which showed that choline abundance was significantly lower in the yolks of eggs that were stored at $5^{\circ} \mathrm{C}$ for five weeks, compared to in the yolks of eggs that were stored at $23^{\circ} \mathrm{C}$ for five weeks.

\section{Conclusion}

This research has shown that storing eggs in refrigerated conditions for five weeks at $5^{\circ} \mathrm{C}$ has a significant impact on the resulting metabolite profile of egg yolk, compared to storing them for the same length of time at $23^{\circ} \mathrm{C}$. One of the compounds found to be significantly lower in abundance in the yolks of eggs stored at $5^{\circ} \mathrm{C}$ compared to those stored at $23^{\circ} \mathrm{C}$ was choline, which has been previously identified as a potential biomarker for egg age as it was found to increase in concentration in egg yolk during an increasing storage period of eggs, at $23^{\circ} \mathrm{C}$. When a targeted study was carried out, in order to observe how choline concentration in egg yolk changes over a twelve-week storage period at $5^{\circ} \mathrm{C}$, it was found that although there was some statistical significance in concentration between some sets of eggs of different ages, there was no real trend in choline concentration that could be established, certainly not any significant increase in concentration over the twelve weeks of storage. These results prove that refrigerating eggs at $5^{\circ} \mathrm{C}$ can prevent the accurate prediction of egg 
age based on choline concentration, and thereby inhibit the use of choline as a biomarker of egg age, capable of detecting cases of fraud due to sell-by and use-by date mislabelling.

\section{Acknowledgements}

This research was supported by Keele University with help and advice contributed by Harper Adams University. Thanks to both The National Institute of Poultry Husbandry and Oaklands Farm Eggs Ltd for providing eggs for this research.

This research did not receive any specific grant from funding agencies in the public, commercial, or not-for-profit sectors.

\section{References}

FDA. (2018). Bioanalytical Method Validation: Guidance for Industry.

Gika, H. G., Theodoridis, G. A., Plumb, R. S., \& Wilson, I. D. (2014). Current practice of liquid chromatography-mass spectrometry in metabolomics and metabonomics. Journal of Pharmaceutical and Biomedical Analysis, 87, 12-25.

https://doi.org/10.1016/j.jpba.2013.06.032

Goodacre, R., Vaidyanathan, S., Dunn, W. B., Harrigan, G. G., \& Kell, D. B. (2004).

Metabolomics by numbers: Acquiring and understanding global metabolite data. Trends in Biotechnology, 22(5), 245-252. https://doi.org/10.1016/j.tibtech.2004.03.007

Hong, E., Lee, Y., Jeong, Y., Park, M., Kim, B. H., \& Sook, H. (2017). Modern analytical methods for the detection of food fraud and adulteration by food category. Journal of the Science of Food and Agriculture, 97, 3877-3896. https://doi.org/10.1002/jsfa.8364

Johnson, A. E., Sidwick, K. L., Pirgozliev, V. R., Edge, A., \& Thompson, D. F. (2018). Metabonomic Profiling of Chicken Eggs during Storage Using High-Performance Liquid Chromatography-Quadrupole Time-of-Flight Mass Spectrometry. Analytical Chemistry, 90, 7489-7494. https://doi.org/10.1021/acs.analchem.8b01031 
Johnson, A. E., Sidwick, K. L., Pirgozliev, V. R., Edge, A., \& Thompson, D. F. (2019). The use of metabonomics to uncover differences between the small molecule profiles of eggs from cage and barn housing systems. Food Control, 100, 165-170. https://doi.org/10.1016/j.foodcont.2019.01.023

Li-Chan, E. C. Y., \& Kim, H. O. (2008). Structure and Chemical Compositions of Eggs. In M. Y (Ed.), Egg Bioscience and Biotechnology (pp. 1-95). Hoboken, NJ USA: John Wiley \& Sons, Inc.

Lockman, P. R., \& Allen, D. D. (2002). The Transport of Choline. Drug Development and Industrial Pharmacy, 28, 749-771. https://doi.org/10.1081/DDC-120005622

Manning, L., \& Soon, J. M. (2014). Developing systems to control food adulteration. Food Policy, 49, 23-32. https://doi.org/10.1016/j.foodpol.2014.06.005

Moco, S., Bino, R. J., De Vos, R. C. H., \& Vervoort, J. (2007). Metabolomics technologies and metabolite identification. Trends in Analytical Chemistry, 26, 855-866. https://doi.org/10.1016/j.trac.2007.08.003

Regulation (EC) 853/2004. (2004). Official Journal of the European Union, L139/55.

Rubert, J., Zachariasova, M., \& Hajslova, J. (2015). Advances in high-resolution mass spectrometry based on metabolomics studies for food - a review. Food Additives \& Contaminants: Part A, 32(10), 1685-1708.

https://doi.org/10.1080/19440049.2015.1084539

Sangster, T., Major, H., Plumb, R., Wilson, A. J., \& Wilson, I. D. (2006). A pragmatic and readily implemented quality control strategy for HPLC-MS and GC-MS-based metabonomic analysis. The Analyst, 131, 1075-1078. https://doi.org/10.1039/b604498k

Spink, J., \& Moyer, D. C. (2011). Defining the Public Health Threat of Food Fraud. Journal of Food Science, 76, 157-163. https://doi.org/10.1111/j.1750-3841.2011.02417.x

Spink, J., Ortega, D. L., Chen, C., \& Wu, F. (2017). Food fraud prevention shifts the food risk 
focus to vulnerability. Trends in Food Science \& Technology, 62, 215-220.

https://doi.org/10.1016/j.tifs.2017.02.012

Tähkäpää, S., Maijala, R., Korkeala, H., \& Nevas, M. (2015). Patterns of food frauds and adulterations reported in the EU rapid alarm system for food and feed and in Finland. Food Control, 47, 175-184. https://doi.org/10.1016/j.foodcont.2014.07.007

Tang, H.-R., \& Wang, Y.-L. (2006). Metabonomics: a Revolution in Progress. Progress in Biochemistry and Biophysics, 33, 401-417.

Theodoridis, G. A., Gika, H. G., Want, E. J., \& Wilson, I. D. (2012). Liquid chromatographymass spectrometry based global metabolite profiling: A review. Analytica Chimica Acta, 711, 7-16. https://doi.org/10.1016/j.aca.2011.09.042

Wang, Q., Jin, G., Wang, N., Guo, X., Jin, Y., \& Ma, M. (2017). Lipolysis and Oxidation of Lipids during Egg Storage at Different Temperatures. Czech Journal of Food Science, 35, 229-235. https://doi.org/10.17221/174/2016-CJFS

Wilson, I. D., Plumb, R., Granger, J., Major, H., Williams, R., \& Lenz, E. M. (2005). HPLCMS-based methods for the study of metabonomics. Journal of Chromatography B: Analytical Technologies in the Biomedical and Life Sciences, 817, 67-76. https://doi.org/10.1016/j.jchromb.2004.07.045

Zeisel, S. H., \& Da Costa, K. A. (2009). Choline: An essential nutrient for public health. Nutrition Reviews, 67, 615-623. https://doi.org/10.1111/j.1753-4887.2009.00246.x 
Table 1. Table showing the mean peak areas for eggs stored at both temperatures, RSD\%s and initial $t$-test $p$-values of 29 compounds found to still show statistical significance in abundance between eggs that were stored at $5^{\circ} \mathrm{C}$ and $23^{\circ} \mathrm{C}$, following confirmatory t-tests based on the raw data. Green upwards arrows indicate a higher compound abundance, and red downwards arrows indicate a lower compound abundance compared to the other storage temperature.

\begin{tabular}{|c|c|c|c|c|c|}
\hline $\begin{array}{c}\text { Feature } \\
m / z \\
+/-<1 p p m\end{array}$ & $\begin{array}{c}\text { Retention } \\
\text { Time median } \\
\text { (mins) }\end{array}$ & $\begin{array}{c}\text { Mean Peak } \\
\text { Area } 23^{\circ} \mathrm{C}\end{array}$ & $\begin{array}{c}\text { Mean Peak } \\
\text { Area } 5^{\circ} \mathrm{C}\end{array}$ & RSD\% & $\begin{array}{c}\text { T-test } \\
\text { p-value }\end{array}$ \\
\hline 104.1072 & 0.79 & $14792632 \uparrow$ & $9382779 \downarrow$ & 1.9 & 0.0038 \\
\hline 165.0544 & 0.86 & $191284 \uparrow$ & $159869 \downarrow$ & 2.8 & 0.0053 \\
\hline 257.2471 & 14.89 & $255184 \uparrow$ & $165207 \downarrow$ & 5.5 & 0.0001 \\
\hline 279.2275 & 19.74 & $706336 \uparrow$ & $454429 \downarrow$ & 5.7 & 0.0025 \\
\hline 283.2980 & 26.38 & $259237 \uparrow$ & $149094 \downarrow$ & 14.3 & 0.0033 \\
\hline 303.2524 & 8.17 & $1320798 \uparrow$ & $914764 \downarrow$ & 3.0 & 0.0036 \\
\hline 331.2856 & 14.88 & $60975406 \uparrow$ & $40877955 \downarrow$ & 2.7 & 0.0002 \\
\hline 359.3172 & 23.67 & $35308950 \uparrow$ & $25512900 \downarrow$ & 2.0 & 0.0048 \\
\hline 367.2789 & 19.10 & $218885 \uparrow$ & $166916 \downarrow$ & 6.2 & 0.0091 \\
\hline 377.2643 & 12.42 & $2313274 \uparrow$ & $1654002 \downarrow$ & 8.7 & 0.0097 \\
\hline 387.3465 & 26.84 & $207381 \uparrow$ & $145533 \downarrow$ & 9.2 & 0.0015 \\
\hline 404.2076 & 1.26 & $1113758 \downarrow$ & $1858424 \uparrow$ & 3.2 & 0.0017 \\
\hline 427.3098 & 13.65 & $168619 \uparrow$ & $56186 \downarrow$ & 13.1 & 0.0004 \\
\hline 438.3448 & 32.57 & $235522 \downarrow$ & $294344 \uparrow$ & 20.2 & 0.0041 \\
\hline 485.3340 & 17.63 & $175814 \uparrow$ & $115321 \downarrow$ & 3.5 & 0.0029 \\
\hline 504.3074 & 17.68 & $971614 \uparrow$ & $697883 \downarrow$ & 2.6 & 0.0025 \\
\hline 517.3000 & 1.63 & $429372 \uparrow$ & $188738 \downarrow$ & 26.0 & 0.0020 \\
\hline 526.2922 & 8.37 & $907058 \uparrow$ & $534077 \downarrow$ & 21.7 & 0.0026 \\
\hline 642.4310 & 28.27 & $14658 \downarrow$ & $247995 \uparrow$ & 12.8 & 0.0003 \\
\hline 658.5427 & 45.23 & $921517 \uparrow$ & $693321 \downarrow$ & 0.6 & 0.0041 \\
\hline 670.4635 & 31.28 & $20424 \downarrow$ & $202309 \uparrow$ & 4.7 & 0.0006 \\
\hline 721.5882 & 45.80 & $194185 \downarrow$ & $330604 \uparrow$ & 5.0 & 0.0078 \\
\hline 774.5572 & 29.22 & $994774 \uparrow$ & $702310 \downarrow$ & 10.4 & 0.0052 \\
\hline 848.7762 & 69.81 & $410909719 \downarrow$ & $478028271 \uparrow$ & 1.8 & 0.0090 \\
\hline 859.6359 & 30.15 & $99628 \downarrow$ & $121827 \uparrow$ & 15.3 & 0.0093 \\
\hline 878.7347 & 68.50 & $2326106 \downarrow$ & $2624913 \uparrow$ & 3.3 & 0.0031 \\
\hline 972.6113 & 10.20 & $164290 \uparrow$ & $122011 \downarrow$ & 2.0 & 0.0090 \\
\hline 985.6246 & 17.66 & $274722 \uparrow$ & $168627 \downarrow$ & 2.6 & 0.0016 \\
\hline 992.8881 & 73.67 & 454001 & $479630 \uparrow$ & 2.3 & 0.0046 \\
\hline
\end{tabular}


